\title{
Implementation and Analysis of AODV Routing Protocol with and without LTE Network
}

\author{
Anita Mashal \\ Dept of Computer Science and \\ Engineering \\ KLSGIT \\ Belagavi, India
}

\author{
Vidya R. Kulkarni \\ Dept of Computer Science and \\ Engineering \\ KLSGIT \\ Belagavi, India
}

\author{
S. A. Kulkarni, PhD \\ Dept of Computer Science and \\ Engineering \\ KLSGIT \\ Belagavi, India
}

\begin{abstract}
LTE stands for Long Term Evolution, which is a standard provided by 3 GPP ( 3 rd Generation Partnership Project) for high peak data rates usage with an uplink and downlink speed of up to $50 \mathrm{Mbps}$ and $150 \mathrm{Mbps}$ respectively. LTE is the next step forward in cellular $3 \mathrm{G}$ services. It is currently the most well known wireless data communication technology for mobile devices. Routing is a specific operation performed to route the packets from the source to destination in any network. Hence there is a requirement of a protocol or algorithm to determine the best way to transfer the data. The paper mainly deals with comparing and analyzing the performance of AODV routing protocol with and without LTE network. The topology used for the analysis of the parameters is same as to the architecture of the LTE network. The different metrics compared and analyzed in this paper are throughput, delay and jitter. The simulation results show that the AODV routing protocol with LTE network provides better results with respect to all the parameters which are analyzed.
\end{abstract}

\section{Keywords}

LTE, 3GPP, AODV.

\section{INTRODUCTION}

Cellular systems will no longer sustain the recent, rapidly increasing demand for applications like VoIP, Internet surfing, online games, etc., due to their limited capacity and data rate. This drove the $3 \mathrm{GPP}$ to produce the LTE cellular system, to achieve higher data rates and capacity to support those multimedia applications. The recent enhance of mobile data treatment and appearance of latest applications has motivated the 3GPP to evolve towards the Long Term Evaluation.LTE is mainly a standard for wireless phones and mobile devices which will connect to the internet. It offers much superior speeds than conventional $3 \mathrm{G}$ technologies. The architecture of this network contains Evolved Packet Core and Radio Access Network. Further EPC is a combination of PGW, MME, SGW and RAN is a collection of user nodes and eNBs [2].The architecture of LTE network is illustrated in Fig 1 .

Functional elements of this architecture contain Evolved nodeBs: are enhanced base transceiver systems which provide the network air interface. Basically these supply the signals to the mobile devices, for instance cell phones, so that they possibly will exchange data with the internet. SGW: It manages and stores User node contexts. It also manages all the data that gets sent to them and performs the necessary actions and replies.MME: It is used User node tracking \& for choosing the SGW for a UE It is also dependable for authenticating the user. PGW: gives connectivity to the User node in the entire networks through the position of exit \& entry point of traffic for the specified User node.

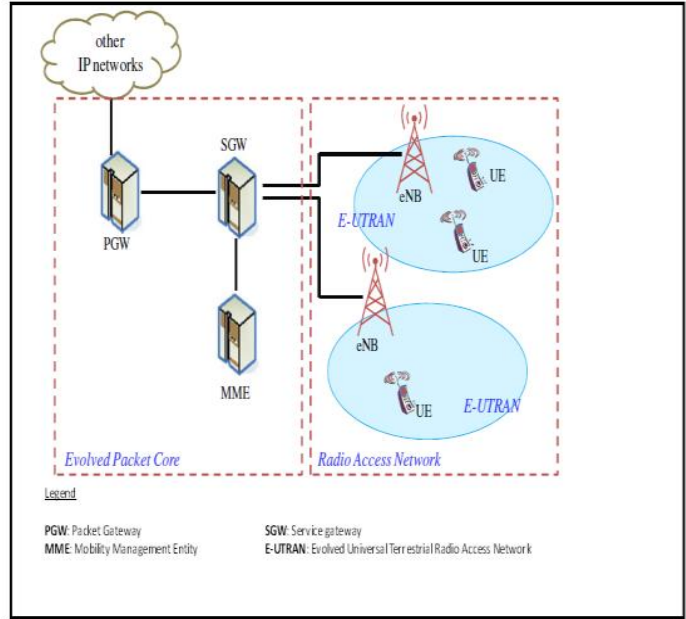

Fig 1: LTE system Architecture

A User node may contain connectivity with multiple PGW for accessing several PDN's [3]. Mobile Ad-hoc Networks are the specific type of wireless network, where mobile nodes are connected through interface creating a temporary network. It is should not contain a fixed infrastructure. Routing is major issue in such networks because of the increasing mobility and dynamic infrastructure of MANETS.

\subsection{MANETs Routing Protocols:}

Motivation for such networks started from military application, where military will never depend on a fixed infrastructure. These networks may not use the wired routing algorithms because of rapid change in the topology. The routing-protocol is necessary when the source requires to communicate with destination. Hence they are needed.

They are differentiated as below, in Fig 2 .

1. Reactive

2. Proactive

3. Hybrid

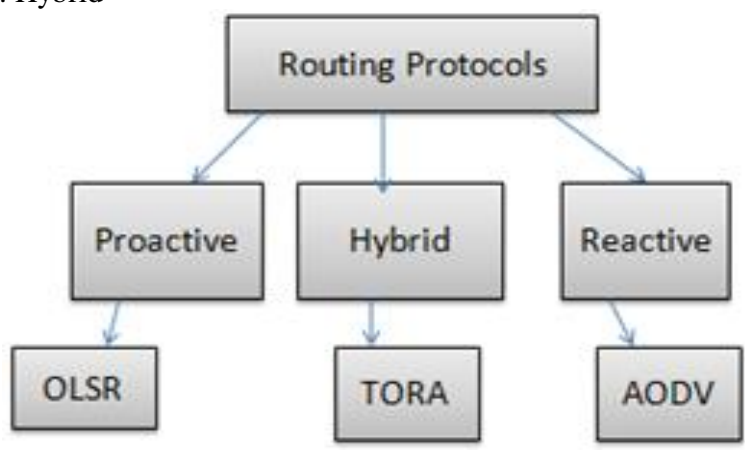

Fig 2: MANETs Routing Protocols 


\subsubsection{Proactive Protocols}

These are fundamentally referred as Table driven type of routing protocols. Specifically all the nodes will contain routing tables, which contains routing information for the entire network. When a new node is entered or removed, to or from the network, control messages are sent to adjacent nodes, later they will update to their routing tables. Even though routing path is not necessary, every node contains path to rest of each single node in the entire network. Examples of some such protocols are OSPF and OLSR.

\subsubsection{Reactive Protocols}

These are called as on demand driven type of protocols. Source discovers the path to the destination only if it has some data to send. Due to the demand, it begins the route discovery, to discover the path to the specific destination node. Later this specified path is used for overall communication e.g. AODV.

\subsubsection{Hybrid Protocols}

These will act as proactive \& reactive protocols together. Firstly it acts as proactive, because in the initial stage nodes include tables. Later when nodes find that they don't comprise of any route to destination node, they start route discovery and behave similar to reactive protocols. TORA and ZRP are hybrid protocols [5].

\section{RELATED WORK}

In paper [7] authors introduce, how the LTE protocol stack operates, it even gives the history of LTE network and provides requirements which will determine the priorities and functions of LTE. It also discusses aspects of this standard such as quality of services, scheduling, control and management functions, and also power operations. In Paper [8] authors describe the study of interactions among the applications, network transport protocol and their impact on performance, with the help of the combination of active and passive measurements. It consists of a new and light weight bandwidth estimation technique for LTE networks. It provides the requirement to build transport-protocol mechanisms and applications which are LTE-friendly. In current years, considering various routing protocols and analyzing their performance has been carried out, in which simulation model contains a constant network-size and a varying pause times. This prescribed work has not taken into account the influence of the mobile nodes' pause time is set constant, but the network size is varying. The authors in paper [9], consider the model with a dynamic network size and an invariable pause time. Based on the routing load and connectivity this paper thoroughly discuses the performance evaluation and comparison of four distinctive routing protocols: AODV, DSDV, DSR, TORA of the Adhoc networks with the various simulation model and metrics, and they provide complete conclusions. Routing is an key issue in the Adhoc-networks and numerous routing protocols have established and proposed such as OLSR, AODV, DSDV, DSR, ZRP, TORA and LAR to enhance the routing performance and reliability. The authors in paper [10] specify the characteristics of these protocols OLSR, AODV and ZRP based on the specific performance metrics like, delay, throughput, jitter and packet delivery ratio by rising number of nodes in the entire network. This study guarantees that OLSR and ZRP perform better in thick or dense networks for low mobility and low traffic. ZRP performs better/well than OLSR and AODV in high mobility and traffic environment.

Adhoc network is an important part for communication in the mobile devices. A cellular network is a collection of mobile nodes which will form topology dynamically, without the existing infrastructure. Routing is a mechanism which transmits the packets to destination through source. Different types of routing protocols are Proactive, Reactive and Hybrid. Reactive or on demand approach is well accepted. In paper [11], authors proceed with an overview of AODV protocols describing their characteristics and functionalities with different parameters like Route Discovery, Route Maintenance, and Flooding and provide their advantages and limitations. The authors also presented the AODV protocol and survey their different security enhancements that are proposed for this protocol with the help of many researchers. In mobile Adhoc infrastructure, mobile nodes can be organized freely. This dynamic connectivity nature of nodes will allow cellular network to be established anywhere/time. Loads of efforts have been done, to provide support \& solution to various problems and challenges related to this network.

\section{PROPOSED APPROACH}

In this current technology, Mobile Ad-Hoc network aim to offer efficient communication with the help of routing functionality. The rapidly increasing technology requires interest in a number of areas such as routing, bandwidth, security, power consumption, simulations for achieving 4 generation environment and resulting better performance. Cellular systems will no longer sustain the recent, rapidly growing demand for applications like VoIP, Internet surfing, online games, etc., due to their limited capacity and data rate. This drove the 3GPP to produce the LTE cellular system, to achieve higher data rates and capacity to support those multimedia applications. This paper focuses at proposing an AODV routing protocol with LTE environment. The comparison of AODV protocol with and without LTE is performed for improvising the throughput and reducing the jitter and delay.

\section{IMPLEMENTATION}

Routing is a major process performed to route the packets through destination from source in any network. Routing protocols are referred to find the route, to transfer the overall data between the nodes within the network. The paper includes.

a. AODV routing protocol with LTE

b. AODV routing protocol without LTE

\section{Ad hoc On-Demand Distance Vector Routing Protocol}

This protocol uses a reactive approach and it uses both unicast and multicast routing: it initiates a path on demand at the beginning of a communication in the entire network and the usage continues until it breaks, later a fresh and new route is initiated. It permits mobile nodes to transfer packets to necessary destination through the usage of neighbor's node which is not capable to communicate directly. It uses routing table, which contains only one entry for every destination. A route replay is transmitted to the source and later, to pass the data packets to the destination depending on the routing table entries in this protocol [12]. Fig 3 defines the basic algorithm of AODV protocol. As the aim is to analyze the performance of AODV protocol with and without LTE network, NS-2 Simulation tool is used. Network Simulator-2 is fundamentally identified as NS2, which is generally an event driven simulation tool which helps in identifying the dynamic nature of network. Topology illustrated in the tool is identical to the architecture of LTE network. Various components in the topology are illustrated as below, Node 0 is the evolved NodeB (eNB) or base station, Node 1 the Serving Gateway 
(S-GW) or Mobility Management Entity (MME), and Node 2 is server. Rest all the nodes are user equipments (UE). The obtained results are efficiently analyzed for below performance metrics: Throughput, delay and Jitter.

The simulation parameters used are Channel typeChannel/wireless channel, Radio Propogation modelPropogational/Two Ray model, Mac type- 802.11, Antenna model- Antenna/Omni Antenna, Interface Queue-LTEQueue, Number of nodes-13, Simulation time-30s.

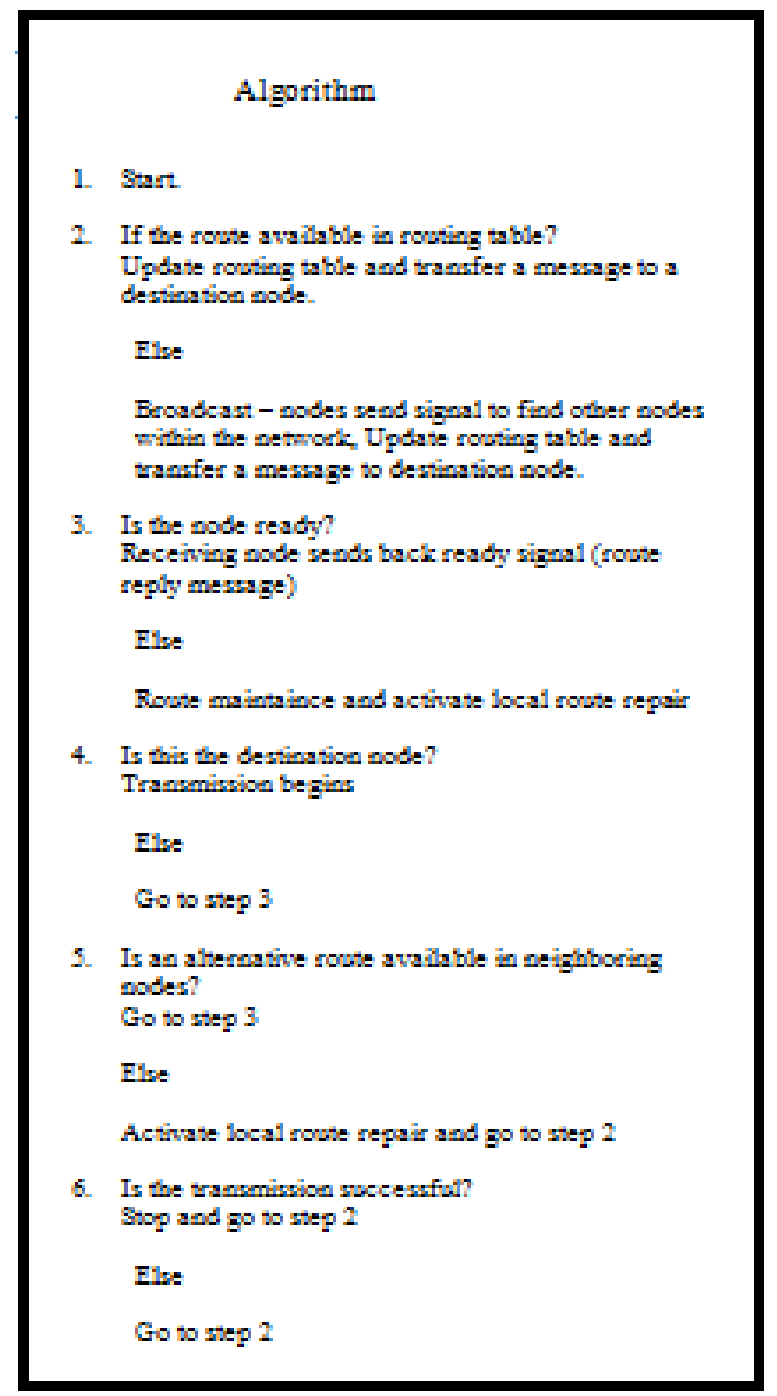

Fig 3: Basic Algorithm of AODV protocol

\section{RESULTS AND DISCUSSIONS}

In this paper the performance of AODV protocol is carried out with and without LTE network. The following are the metrics used for the comparision of this protocol with and without LTE network. All these specified metrics are considered and calculated from the trace file generated after the successful completion of simulation. After getting the metrics, graphs are plotted.

\subsection{Throughput}

Throughput is basically referred as the total packets delivered to the destination from the source successfully within the given time.

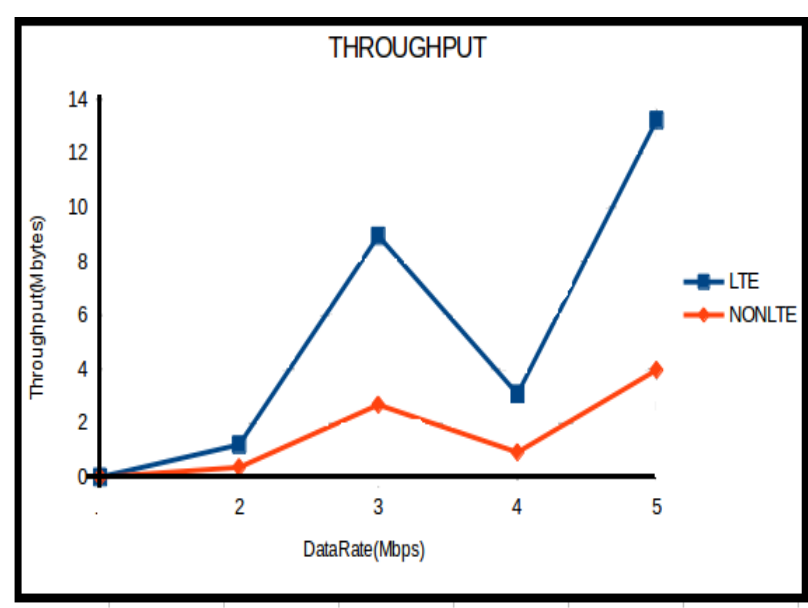

Fig 4: Comparison graph for throughput

Above Fig 4 describes the relationship between AODV protocol with and without LTE Network with respect to throughput metric. By considering the above graph, we can view that, throughput of ADOV with LTE Network increases efficiently. The graph consists of two axis, wherein $\mathrm{x}$-axis presents the data rate which is represented in Mbps and y-axis presents the throughput which is represented in MBytes. Observation clarifies that throughput increases rapidly with respect to data rate for AODV protocol with LTE when compared to AODV protocol without LTE.

\subsection{Delay}

Delay is fundamentally referred as the total time used by a packet, which is used to transmit from its source to destination. Calculation of delay is carried out for every packet in the defined time interval.

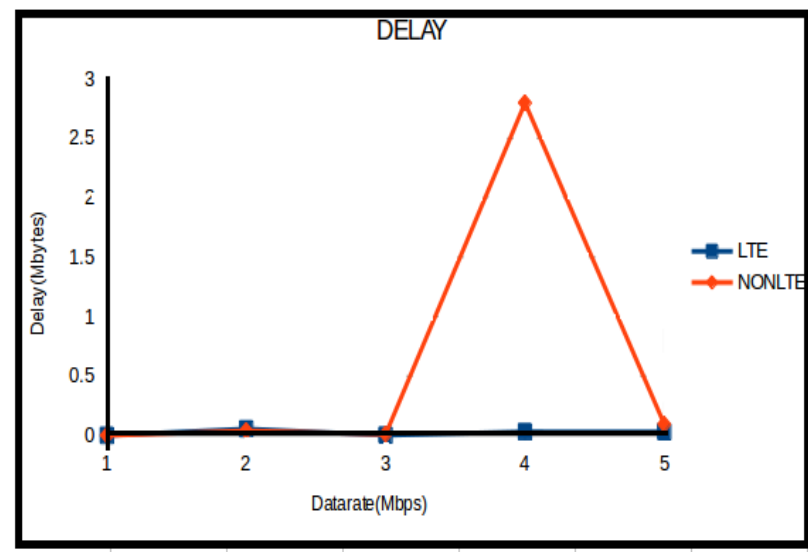

Fig 5: Comparison graph for Delay

Fig 5 represents the relationship between AODV protocol with and without LTE Network according to delay as a metric. $X$ - axis refers the data rate which is represented in Mbps and Y - axis refers the delay which is represented in MBytes. By referring the above graph, it is very clear that the total amount of time taken to transfer the packets is lower for ADOV with LTE when compared to AODV without LTE Network.

\subsection{Jitter}

Jitter is expressed as the difference in time taken for the number of packets received in the network. The source node will be routing the packets in a continuous manner to the destination node, but destination node may face some 
problems to receive the packets continuously due to traffic in the network. Fig 6 describes the relationship between AODV protocol with and without LTE Network with jitter as a metric. $X$ axis refers the data rate which is represented in Mbps and $\mathrm{Y}$ axis refers the jitter which is represented in MBytes. From above graph, it justifies that the user node has a lesser delay while receiving the packets for ADOV protocol with LTE Network.

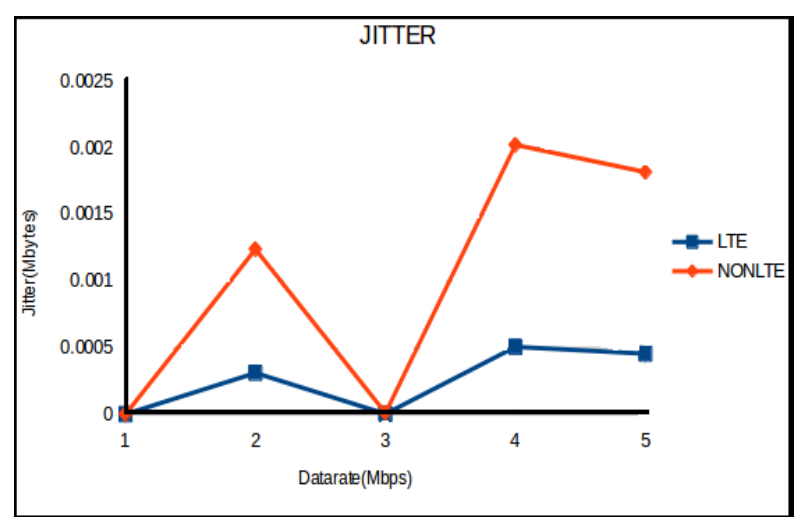

Fig 6: Comparison graph for Jitter

The performance of the AODV Routing Protocol with LTE Network is better with respect to all the metrics which are measured and compared to the AODV Routing Protocol without LTE.

\section{CONCLUSION}

In this prescribed paper an attempt is made to route the packets through AODV routing protocol and compare the performance with and without LTE Network. Based on the results displayed through simulation, the proposed work justifies that AODV routing protocol achieves better performs with LTE network in terms of throughput, delay and jitter.

\section{FUTURE WORK}

In future an attempt can be made to analyze the performance of other routing protocols including OLSR, DSR with LTE network and the performance of AODV routing protocol with LTE can be analyzed using other performance metrics like packet loss ratio, routing overhead. Even the comparison of two different routing protocols can be done with LTE network.

\section{REFERENCES}

[1] Tejaswini G N , Kartikeya Rao G V , Manjunath P S , Rajeshwari Hegde, Research gate conference paper, sep 2014 "Comprehensive analysis of routing protocols for the application layer of LTE”.Ding, W. and Marchionini, G. 1997 A Study on Video Browsing Strategies. Technical Report. University of Maryland at College Park.

[2] "Long Term Evolution Protocol Overview White paper" October 2008.

[3] "Technical White paper Long Term Evolution: A Technical Overview".

[4] F. Capozzi, G. Piro, L.A. Grieco, G. Boggia, and P. Camarda "Downlink Packet Scheduling in LTE Cellular Networks: Key Design Issues and a Survey".
[5] Manjeet Gupta and Sonam Kaushik, International Journal of Computational Engineering Research, June 2012, "Performance Comparison Study of AODV, OLSR and TORA Routing Protocols for MANETS".

[6] Azzedine Boukerche, Begumhan Turgut, Nevin Aydin, Mohammad Z. Ahmad, Ladislau Boloni, and

[7] Freescale Semiconductors, (2008) "Long Term Evolution Protocol Overview"

[8] Junxian Huang, Feng Qian, YihuaGuo, Yuanyuan Zhou, QiangXu, Z. Morley Mao, Subhabrata Sen, Oliver Spatscheck, "An In-depth Study of LTE: Effect of Network Protocol and Application Behavior on Performance", In Proceedings of the ACM Special Interest Group on Data Communication (SIGCOMM), 2013.

[9] Li Layuan , Li Chunlin, Yaun Peiyan, "Performance evaluation and simulations of routing protocols in ad hoc networks", Elsevier 2007

[10] Hrituparna Paul Priyanka Sarkar "A Study And Comparison of Olsr, Aodv And Zrp Routing Protocols In Ad Hoc Networks", International Journal of Research in Engineering and Technology, Aug-2013.

[11] Rahul Patel, Anjuman Ranavadiya, Shreya Patel, "A Survey Paper on AODV Routing Protocol for MANET", International Journal for Scientific Research \& Development, 2014.

[12] Devideen ahirwar, Prof. Sarvesh Singh Rai, "Improvement of AODV Routing Protocol Algorithm with Link Stability and Energy Efficient Routing for MANET", International Journal of Computer Science Engineering and Technology ( IJCSET), Feb-2014

[13] Deeksha Bhardwaj, Shruti sekar, "Adhoc Routing Protocol: A Tutorial", IJACKD Journal Of Research, Sep-2014

[14] Aastha Kohli, Sukhbir, "A Review Paper On Routing Protocol Comparison", International Journal for Research and Applied Science And Engineering Technology, Sep-2013

[15] Prashant Kumar, Maurya , Gaurav Sharma, Vaishali Sahu, Ashish Roberts, Mahendra Srivastav, "An Overview Of AODV Routing Protocol ," International Journal of Modern Engineering Research, 2012.

[16] Neha Singh, Prof. Rajeshwar Lal Dua, Vinita Mathur,“ Network Simulator NS2-2.35", International Journal of Advanced Research in Computer Science and Software Engineering, May-2012

[17] Genita Gautam, Biswaraj Sen, "Design and Simulation of Wireless Sensor Network in NS2", International Journal of Computer Applications, March-2015.

[18] Pallavi S. Katkar, Dr. Vijay R. Ghorpade, "Comparative Study of Network Simulator: NS2 and NS3", International Journal of Advanced Research in Computer Science and Software Engineering, March-2016. 